\title{
Multichannel interferometer system for the helically symmetric experiment
}

\author{
D. L. Brower, ${ }^{\text {a) }}$ C. Deng, and W. X. Ding \\ Electrical Engineering Department, and Institute of Plasma and Fusion Research, University of California \\ at Los Angeles, Los Angeles, California 90095-1594 \\ D. T. Anderson and W. Mason \\ Electrical Engineering Department, University of Wisconsin at Madison, Madison, Wisconsin 53706
}

(Presented on 21 June 2000)

\begin{abstract}
A multichannel millimeter-wave interferometer system has been designed, fabricated and installed on the helically symmetric experiment (HSX), located at the University of Wisconsin, Madison. The interferometer system will view the plasma cross section along nine adjacent chords with $1.5 \mathrm{~cm}$ spacing. With this arrangement, coverage will span from the low-field side plasma scrape-off layer to well past the magnetic axis. For the plasma densities anticipated on HSX, a solid-state source operating at $288 \mathrm{GHz}$ will be utilized. At this frequency refraction will be manageable, being less than the channel spacing. The source will be bias-tuned and modulated with a sawtooth wave form at $750 \mathrm{kHz}$ in order to generate the intermediate frequency necessary for the heterodyne detection scheme. The signals will be measured using Schottky-diode corner-cube mixers. The interferometer will have sensitivity $n_{e} d l \approx 8 \times 10^{11} \mathrm{~cm}^{-2}$, being able to measure density changes $<1 \%$. Initially, the phase will be evaluated using analog electronics with bandwidth $<10 \mathrm{kHz}$ providing real-time line-integrated output. A digital phase comparator scheme will also be implemented whereby the measured wave forms are directly digitized and the phase evaluated using a software-based algorithm. This will increase the time response up to the modulation frequency of $750 \mathrm{kHz}$. Improved time response will permit measurement of high-frequency density fluctuations along with "fast changes in" the equilibrium profile. (C) 2001 American Institute of Physics.

[DOI: $10.1063 / 1.1319366]$
\end{abstract}

\section{INTRODUCTION}

The helically symmetric experiment (HSX) device is a new concept in toroidal stellarators whose construction was recently completed at the University of WisconsinMadison. This unique device has a quasihelically symmetric magnetic configuration leading to virtually no toroidal curvature in the magnetic field lines responsible for plasma confinement. With aspect ratio of approximately 8, HSX will have the same toroidal curvature as a conventional toroidal device with an aspect ratio of 400. HSX has an axis of symmetry that results in improved single particle confinement as compared to conventional stellarators and will bridge the gap between asymmetric currentless stellarators and symmetric current-driven tokamaks. The goal of the experimental program is to test the improved confinement properties of this device and to advance the stellarator as a fusion reactor. As part of the HSX experimental program, high-resolution diagnostics are required to accurately assess various plasma properties. UCLA, in collaboration with the University of Wisconsin-Madison, is contributing to the HSX experimental program by developing high-resolution density profile and fluctuation diagnostics to determine the electron density distribution and turbulence characteristics; both spatially and temporally. To this end, a high-resolution, multichannel interferometer system, designed and fabricated by UCLA, has recently been installed on the HSX device. The HSX inter-

${ }^{a)}$ Electronic mail: brower@mail.utexas.edu ferometer system bares similarities to previous systems developed by UCLA and employed on TEXT, ${ }^{1}$ MTX, ${ }^{2}$ and MST. ${ }^{3,4}$ This system is currently undergoing testing and calibration in anticipation of HSX startup operations. By highresolution interferometry we mean (1) many spatial samples or chords to resolve small scale structures, (2) fast time response to monitor fast density changes associated with internal instabilities or externally imposed perturbations, and (3) low phase noise to be able to detect small amplitude density changes. Density perturbations in the form of modulated gas feed, power deposition, or internal instabilities can provide information on the electron particle transport coefficients. A high-speed interferometer can also be employed to look at high-frequency electrostatic density fluctuations associated with turbulence and anomalous transport, as is presently being done with the interferometer system on $\mathrm{MST}^{4}$

\section{HSX INTERFEROMETER SYSTEM}

HSX is a toroidal device with major radius $R=1.2 \mathrm{~m}$, average minor radius $\langle a\rangle=15 \mathrm{~cm}$, toroidal magnetic field $B_{T}=1 \mathrm{~T}$, anticipated central electron temperature $T_{e}$ $=1 \mathrm{keV}$, and central electron density $n_{e}<1 \times 10^{13} \mathrm{~cm}^{-3}$ for $200 \mathrm{~kW}$ heating with electron cyclotron resonance heating $(\mathrm{ECRH})$ at $28 \mathrm{GHz}$.

The high-resolution HSX interferometer system consists of nine chords, with $1.5 \mathrm{~cm}$ spacing (and width), covering $13.5 \mathrm{~cm}$ for a given HSX discharge. Slotted ports, with dimensions $13.5 \mathrm{~cm} \times 3.25 \mathrm{~cm}$ at both the machine top and 


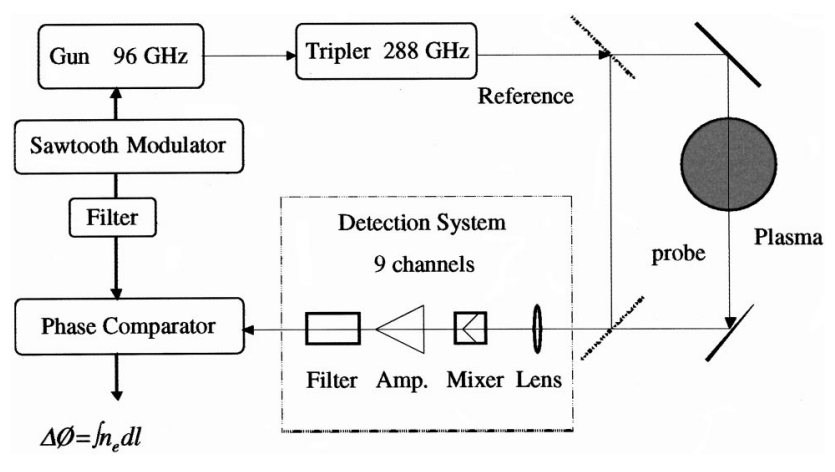

FIG. 1. Block Diagram of HSX interferometer.

bottom, allow this arrangement which will cover from the low-field side scrape-off layer (SOL) to well past the magnetic axis (seven channels from SOL to magnetic axis). The interferometer system will be physically located at one of the four box ports where the low-field side is the larger major radius plasma edge, like the tokamak.

As opposed to using a twin-frequency far-infrared (FIR) laser for the source, as was done on TEXT, MTX, and MST, for the lower-density plasmas anticipated on HSX we will utilize a frequency-modulated solid-state source operating at $288 \mathrm{GHz}$ (approximately $1 \mathrm{~mm}$ ). A solid-state source is less expensive and simpler to operate in comparison with a FIR laser. At this frequency, refraction is expected to be manageable (14 mrad maximum for parabolic density profile), being much less than the channel width. For initial operations, a varactor-tunable Gunn oscillator operating at $72 \mathrm{GHz}$ serves as the input to a system of two doublers providing approximately $0.7 \mathrm{~mW}$ output at $288 \mathrm{GHz}$. The Gunn diode output is varied nominally $50 \mathrm{MHz}$ in $1 \mu$ s with a sawtooth wave form which sweeps the doubler output frequency up to $200 \mathrm{MHz}$. This source is only capable of biasing up to five mixers. A high-power source will be implemented for full nine-channel operation. The high-power source consists of a $96 \mathrm{GHz}$ biastuned Gunn oscillator and an InP tripler which will generate up to $8 \mathrm{~mW}$ of output power at $288 \mathrm{GHz}$. A major advantage of using a solid-state source in the interferometer system is that the high-frequency multiplier is insensitive to feedback and therefore eliminates the need for an isolator, which is sensitive to magnetic fields and introduces signal loss. The multiplier also reduces the frequency sweep requirements for the Gunn oscillator.

The basic technique for an interferometer employing a frequency-modulated source is shown in Fig. 1. The source radiation propagates along two paths to the receiver, one through the plasma (probe path) and the other through free space (reference path). The two paths differ in length $\Delta L$.

The intermediate frequency (IF) signal (after the mixer) is

$C \cos (\Omega t-\Delta \phi)$,

where $\Omega=k \Delta L / c$. The IF frequency is set by varying the path length difference $\Delta L$ or the slope of the sawtooth $k$ $(=\Delta f / \Delta t)$. For a sawtooth period of $1.33 \mu \mathrm{s}$ with $\Delta f$ $=75 \mathrm{MHz}$ and $\Delta L=400 \mathrm{~cm}$, we obtain $\Omega=750 \mathrm{kHz}$, the nominal IF to be used for the HSX interferometer system. The inputs to the phase comparator, either analog or digital, are then the sawtooth-modulated wave form (appropriately filtered) and $C \cos (\Omega t-\Delta \phi)$ with the output being proportional to $\Delta \phi(t)$, the line-averaged density.

In order to generate a multichord system, both the signal and local oscillator beams are expanded in one dimension via parabolic cylindrical mirrors. This provides maximum access to the plasma cross section viewable through the ports as shown in Fig. 2, where a schematic of the diagnostic layout is provided. Quasi-optical beam propagation is utilized with Ni-plated wire mesh beam splitters and collimating optics (high-density polyethylene lens). Overmoded dielectric waveguide is employed for transporting the beam long distances while maintaining beam mode and size. High spatial sampling is achieved by arranging corner-cube GaAs Schottky-diode mixers in a linear array (nine channels maximum) with spacing of $1.5 \mathrm{~cm}$. A focusing lens of width 1.5 $\mathrm{cm}$ samples a portion of the probing beam for each chord.

The optical system is attached to a $2.5 \mathrm{~m}$ tall, 2 ton reaction mass. The reaction mass has been designed to operate with resonant frequency $<30 \mathrm{~Hz}$. The reaction mass is constructed of 5 in. $\times 10$ in. stainless steal tubes filled with sand. These tubes are bolted to building structural I beams which are independent of the floor which holds the HSX device. The reaction mass was created to minimize equipment vibration that contributes to interferometer phase noise. The entire structure is situated on adjustable mounts to facilitate alignment to the HSX vacuum vessel ports.

During initial plasma operations, tests will be made of dichroic filters mounted on the input and exit ports of the HSX vacuum vessel. The initial plasma breakdown and all plasma heating for HSX comes from a $200 \mathrm{~kW}, 28 \mathrm{GHz}$ gyrotron. This radiation and possible harmonic output pose a serious threat to the interferometer system as emission of only a milliwatt along the system optical axis can contaminate the signal. To remedy this problem, dichroic filters (also called perforated plate filters), which act as high-pass filters, are mounted over the polyethylene vacuum windows. The dichroic filters consist of a 0.2 -cm-thick aluminum plate with $0.04 \mathrm{~cm}$ radius holes packed in a hexagonal pattern with $0.015 \mathrm{~cm}$ wall thickness between adjacent holes.

The attenuation that can be achieved is estimated using the standard evanescant propagation equation for cylindrical waveguide. For a cylindrical waveguide of radius $a$, the cutoff wavelength is $\lambda_{c}=3.41 a$, and the power attenuation is given by

$$
P_{\text {trans }}=P_{\text {in }} e^{-\alpha t},
$$

where $t$ is the plate thickness and

$$
\alpha=k_{c}\left[1-\left(\lambda_{c} / \lambda\right)^{2}\right]^{1 / 2} \text {. }
$$

For the hole radius given above, the cutoff frequency is $220 \mathrm{GHz}$ with an estimated attenuation ranging from $92 \mathrm{db}$ at $28 \mathrm{GHz}$ to $68 \mathrm{db}$ at $150 \mathrm{GHz}$. A characteristic of the dichroic filter is that transmission is very good, approaching $100 \%$, at frequencies just above the cutoff. Laboratory tests have shown that approximately $10 \%$ of the signal is lost upon transmission of the $288 \mathrm{GHz}$ source radiation through the two filters. 


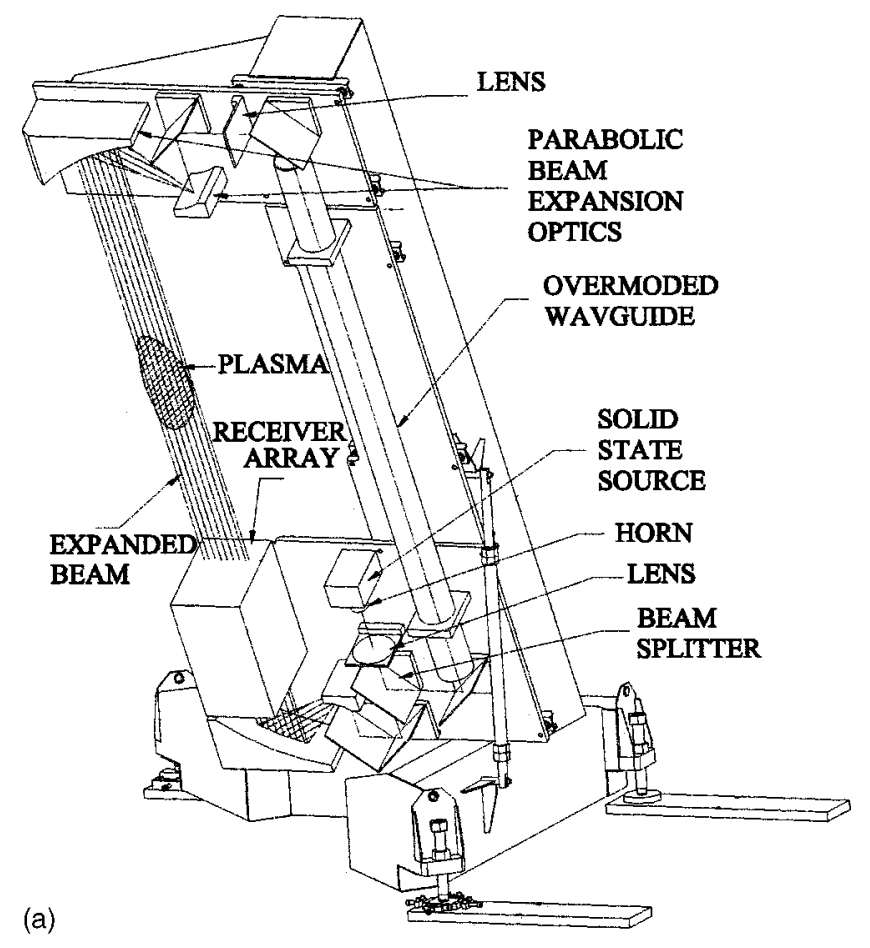

(a)

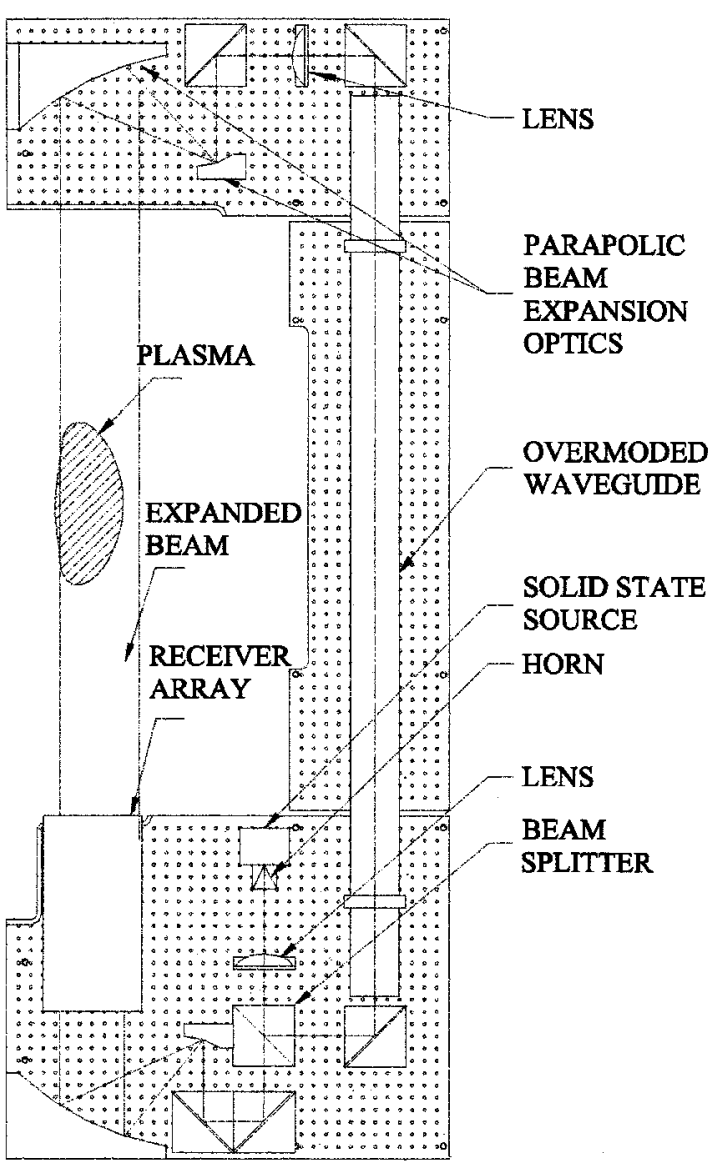

FIG. 2. (a) Three-dimensional plot of interferometer system designed for HSX. (b) Schematic of HSX interferometer system optical layout.

\section{PHASE DETECTION, DATA ACQUISITION, AND SIGNAL PROCESSING}

The phase information from each interferometer chord will be extracted in two ways. First, analog phase detection

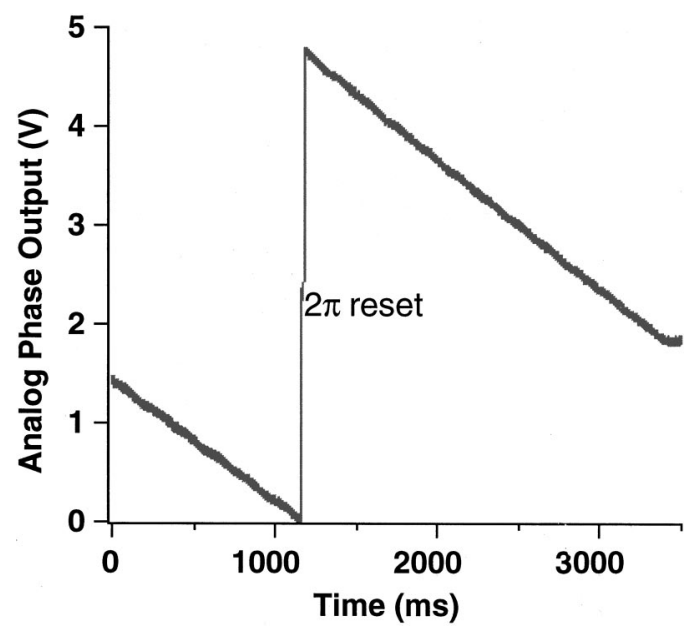

FIG. 3. Analog phase comparator calibration plot. Voltage change going from minimum to maximum corresponds to two phase change.

electronics will be used to provide real-time output from each channel. Analog phase measurement techniques provide a time response of approximately 100-200 $\mu$ s. These data can be used to determine the equilibrium profile and its evolution throughout the plasma discharge. Second, implementation of digital techniques can be employed to reduce the time response to a few microseconds. ${ }^{5}$ In this approach, the filtered reference and signal wave forms at $750 \mathrm{kHz}$ are directly digitized and the phase information extracted via use of software-based algorithms. This is referred to as the digital phase comparator (DPC), which is capable of achieving 4 $\mu$ s time response.

At present, the interferometer hardware has been installed and is being tested on HSX. Preliminary calibration measurements of the interferometer are underway. A plot of the phase output from the analog phase comparator for one of the chords is shown in Fig. 3. For this test, a direct current motor was used to move a mirror and change the path length in the signal leg of the system thereby inducing a phase delay with respect to the reference. The analog phase comparator is designed to reset every $2 \pi$ (or $20 \pi$ ) radians change in phase. For this particular phase comparator channel, the calibration is $2 \pi$ radians $=4.73 \mathrm{~V}$. The measured phase relates to the line-averaged density via the equation

$$
\int n_{e}(z) d z=2.45 \times 10^{12} \Delta \phi(\mathrm{V}) \mathrm{cm}^{-2} .
$$

These measurements indicate that the root-mean-square (rms) phase noise for each channel using the analog phase comparator is approximately $0.024 \mathrm{rad}$ or about $1.6^{\circ}$. This implies a minimum line-averaged density measurement capability of $n_{e} d l=8 \times 10^{11} \mathrm{~cm}^{-2}$. For the central chord of HSX, this would correspond to a density of approximately $2 \times 10^{10} \mathrm{~cm}^{-3}$, less than $1 \%$ of the anticipated central density. This resolution will be sufficient to investigate smallamplitude density fluctuations.

As mentioned earlier, the interferometer bandwidth can be increased through use of the DPC technique. ${ }^{5}$ In Figs. 4(a) and 4(b), the time trace and Fourier transform of the filtered signal wave form is shown for a HSX interferometer chord. 
(a)

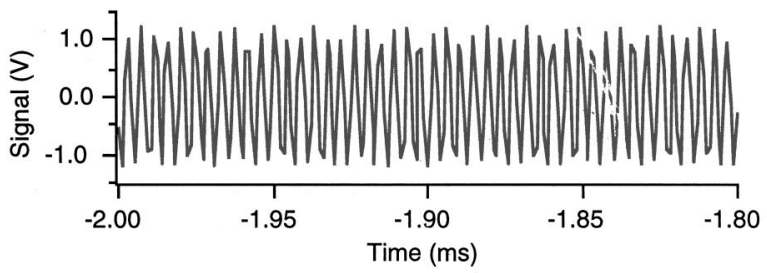

(b)

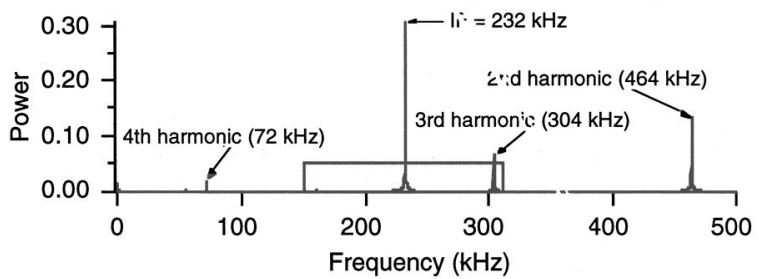

(c)

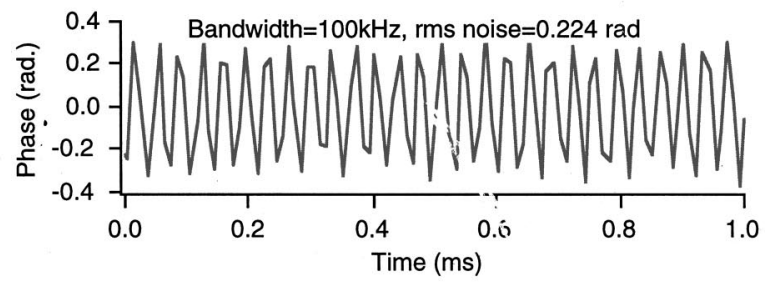

(d)

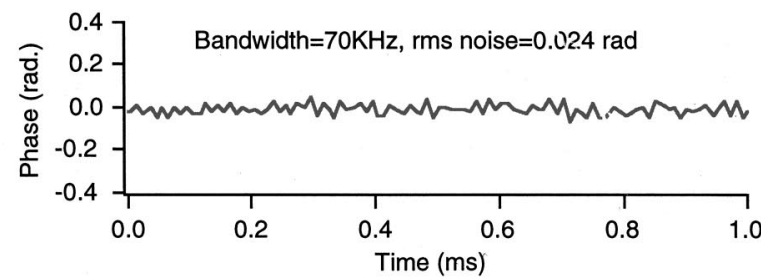

FIG. 4. (a) Time trace of aliased $768 \mathrm{kHz}$ IF signal. (b) Frequency spectrum of the aliased signal showing harmonics. (c) DPC phase noise for $100 \mathrm{kHz}$ bandwidth. (d) DPC phase noise for $70 \mathrm{kHz}$ bandwidth.

The frequency spectra is similar for all the interferometer chords as well as the reference. For a $768 \mathrm{kHz}$ IF frequency with sampling at $1 \mathrm{MHz}\left(f_{\text {Nyquist }}=500 \mathrm{kHz}\right)$, we observe the IF aliased to $232 \mathrm{kHz}$ along with aliased harmonics at 464 $\mathrm{kHz}$ (2nd), $304 \mathrm{kHz}$ (3rd), and $72 \mathrm{kHz}$ (4th). These harmonics persist despite filtering and have a significant impact on the system noise. This can be seen in Figs. 4(c) and 4(d), where the phase noise is plotted for DPC system bandwidths of 100 and $70 \mathrm{kHz}$, respectively. In the DPC software, prior to evaluating the phase, the reference and signal datasets are filtered which determines the system bandwidth. (Typically this bandwidth is maximized by choosing the smaller of the difference between the aliased IF signal and the Nyquist or zero frequency. ${ }^{5}$ ) As shown in the Fig. 4(b), if the bandwidth of this filter is set such that one of the harmonics is included $(\mathrm{bw}=100 \mathrm{kHz}$ ), the system noise level is $0.2 \mathrm{rad} \mathrm{rms}$, a level much higher that the analog phase comparator output. However, with the bandwidth reduced to $70 \mathrm{kHz}$, all harmonics are excluded and the rms phase noise is reduced to $0.024 \mathrm{rad}$. This is the same noise level as the analog phase comparator while increasing the bandwidth by an order of magnitude. To optimize the system for $1 \mathrm{MHz}$ sampling, the IF should ideally be set to $775 \mathrm{kHz}$ as this would permit a maximum bandwidth [i.e., harmonics filtered out] of $100 \mathrm{kHz}$. If the sample speed is increased to 2(5) $\mathrm{MHz}$, the interferometer bandwidth for the DPC can reach $250(750) \mathrm{kHz}$ for an IF set to $750 \mathrm{kHz}$. The need to increase the interferometer bandwidth on HSX will be determined by the measured density fluctuation spectrum. The IF must be kept in the 600-900 $\mathrm{kHz}$ range of frequencies in order for the analog phase comparator to function.

On HSX, a pc based data acquisition system is being employed. For the analog phase comparator, nine channels of phase output will be stored for approximately $100 \mathrm{~ms}$ with $50 \mathrm{kHz}$ sampling. For use of the DPC, ten channels of data acquisition are required with $1 \mathrm{MHz}$ sampling. The phase information from the DPC can easily be computed between HSX discharges.

\section{DENSITY FLUCTUATION MEASUREMENTS}

The good phase resolution coupled with the fast time response of the DPC will permit use of the interferometer as a diagnostic to measure plasma density fluctuations as well as the equilibrium profile. This capability is similar to that presently being exploited on the MST interferometer system. ${ }^{4}$ The sensitivity of the interferometer measurement to fluctuation wave number is determined by the shape and orientation of the sample volume (chord width $1.5 \mathrm{~cm}$ ) with respect to the turbulent modes or structures. A vanishingly small sample volume would be sensitive to all wave numbers. An upper bound on the sensitivity to wave number in a particular direction can be defined when the sample volume length in that direction is half a wavelength, i.e., $k_{\max }$ $=2 \pi / \lambda_{\max }=\pi / L$, where $L$ is the sample volume dimension. For the HSX interferometer chords, in a direction transverse to the beam, the wave number limit is $k_{\max }=2.1 \mathrm{~cm}^{-1}$, while along the beam the upper bound is $0.07 \mathrm{~cm}^{-1}$ for the longest chord. So in the direction perpendicular to the beam, turbulent fluctuations with wavelength greater than $3 \mathrm{~cm}$ can be measured.

\section{CONCLUSION}

The multichannel interferometer system has been installed and is now ready for HSX plasma operations. Calibration has been completed and indicates that the system is capable of measuring small-amplitude high-frequency density fluctuations. Implementation of the high-power source is required to realize the full nine channel capability.

\section{ACKNOWLEDGMENT}

This work is supported by DOE under Grant No. DEFG03-86ER-53225, Task III.

${ }^{1}$ S. K. Kim, D. L. Brower, W. A. Peebles, and N. C. Luhmann, Jr., Rev. Sci. Instrum. 59, 1550 (1988).

${ }^{2}$ B. W. Rice, Rev. Sci. Instrum. 63, 5002 (1992).

${ }^{3}$ S. R. Burns, W. A. Peebles, D. Holly, and T. Lovell, Rev. Sci. Instrum. 63, 4993 (1992).

${ }^{4}$ Y. Jiang, D. L. Brower, and N. E. Lanier, Rev. Sci. Instrum. 70, 703 (1999).

${ }^{5}$ Y. Jiang, D. L. Brower, L. Zeng, and J. Howard, Rev. Sci. Instrum. 68, 902 (1997). 